\title{
Investigation of the substrate influence on the fluorination with Deoxofluor of some highly functionalized alicyclic scaffolds
}

\section{Melinda Nonn, ${ }^{1}$ Ferenc Fülöp, ${ }^{1,2}$ Loránd Kiss, ${ }^{2 *}$}

${ }^{1}$ MTA-SZTE Stereochemistry Research Group, Hungarian Academy of Sciences, H-6720 Szeged, Eötvös u. 6, Hungary

${ }^{2}$ Institute of Pharmaceutical Chemistry, University of Szeged, H-6720 Szeged, Eötvös u. 6, Hungary

Email: kiss.lorand00@gmail.com

kiss.lorand@pharm.u-szeged.hu

\section{Graphical abstract:}

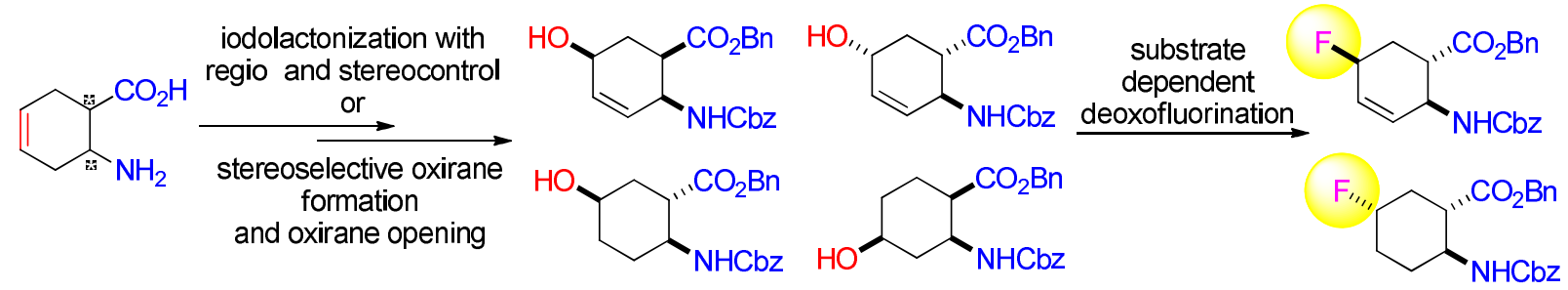

\begin{abstract}
:
Substrate influence on the fluorination of some highly functionalized alicyclic scaffolds with Deoxofluor has been investigated. The selected model compounds, hydroxylated, saturated and unsaturated six-memberd cyclic $\beta$-amino esters, have been synthesized from unsaturated cyclohexane $\beta$-amino acid stereoisomers through iodolactonization with stereo- and regiocontrol or stereoselective ring olefinic bond oxidation followed by regioselective oxirane opening. Depending on the stereostructure of the hydroxylated amino esters, their nucleophilic fluorination under various conditions afforded either the corresponding fluorine-containing substitution or elimination products.
\end{abstract}

Keywords: $\beta$-amino acids, regiocontrol, stereocontrol, fluorination, functionalization Introduction 
Because of the increasing importance of fluorinated molecules in medicinal chemistry [1], the development of selective and controlled efficient fluorination procedures has been a hot topic of synthetic organic chemistry in recent years. This interest is well illustrated by the ever-increasing number of various synthetic publications on this area and related recent reviews [2].

Among the varied fluorination protocols, nucleophilic fluorination through deoxofluorination of organic substrates with commercially available organic fluorinating agents (e.g. DAST, Deoxofluor, Fluolead, XtalFluor) occupies an important segment of fluorination procedures of organic molecules. According to the widely accepted approach, substitution of fluorine for a hydroxy group takes place characteristically through inversion [3]. Deoxofluorination involving hydroxy-fluorine exchange is an unequivocally simple and efficient protocol for the introduction of a fluorine into an organic molecule. However, regio- and stereocontrol as well as stereoselectivity and substrate directing effects remain a challenge when working with highlyfunctionalized scaffolds. During our earlier experimental investigations on various functionalized alicyclic molecules, we have found that these transformations are highly substrate dependent. In addition, the stereochemistry of the substrates as well as the nature of the protecting groups were shown to play a crucial role in the outcome of various fluorinations [4].

\section{Results and Discussion}

The goal of this study was to continue and extend our earlier studies of fluorination of various cyclic saturated or unsaturated $\beta$-amino esters. Therefore, because of their pharmaceutical importance [5], we have selected some $\beta$-amino acid model compounds and intended to collect useful pieces of information about their reactivity and chemical behavior. Specifically, we wanted to explore the regio- and stereochemistry of their transformations and the effect of carboxylic and amino protecting groups (e.g. benzyl and benzyloxycarbonyl).

First, the selective hydroxylation of the ring olefinic bond in cyclohexene cis $\beta$-amino acid $( \pm)-1$ was accomplished through regio- and stereoselective iodolactonization. Resulting lactone $( \pm)-2$ [6] furnished, after HI elimination with diazabicycloundecene (DBU), unsaturated bicyclic lactone ( \pm )-3. Lactone opening in $( \pm)-3$ with sodium benzyl alcoholate $(\mathrm{BnONa})$ at $50{ }^{\circ} \mathrm{C}$ led to all-cis hydroxylated amino ester $( \pm)-\mathbf{4}$, bearing the hydroxy group at C-5 (Scheme 1). Next, this all-cis product was subjected to fluorination via hydroxy-fluorine exchange under a series of experimental conditions by varying the temperature and solvents, with the aid of commercially 
available nucleophilic fluorinating agents. However, contrary to our expectations [6c], the reaction afforded only an unidentifiable mixture of compounds with a large amount of polymeric material (Scheme 1). The failure of the fluorination of $( \pm)-4$ might be explained by stereochemical factors. Namely, it is surmised that the fluorinating agent was not able to transform the hydroxy function into the corresponding good leaving group because of the steric hindrance of the bulky benzyl ester and carbamate moieties.

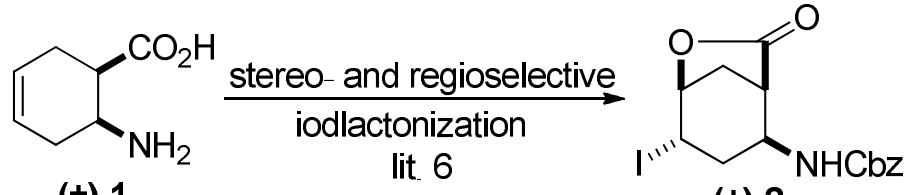

$( \pm)-1$ lit 6

(士)-2

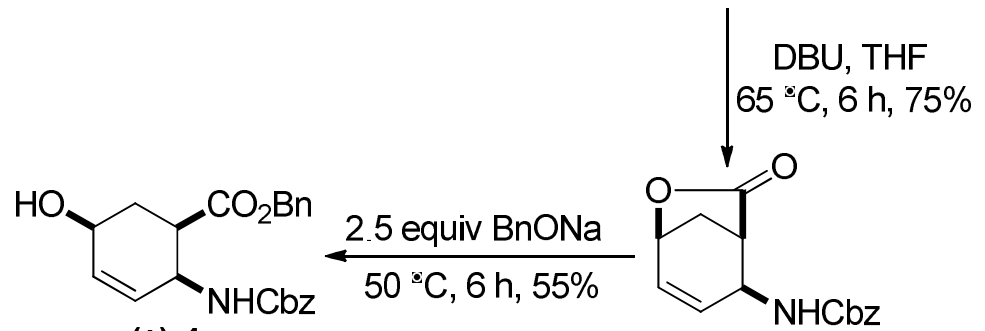

(士) 4

$( \pm)-3$

Scheme 1.

This failure did not deter us to continue our experimental investigation with other benzyl/benzyloxycarbonyl protected isomers of cyclohexene amino acids. It was an obvious necessity to change the stereochemical arrangement of the functional groups in the starting material. Thus, cyclohexene trans amino acid $( \pm)-5$ was converted with selective and stereocontrolled iodolactonization to the corresponding lactone ( \pm )-6 [6]. Analogously to its cis counterpart, HI elimination with DBU gave unsaturated lactone $( \pm)-7$ and subsequent opening of the heteroring with $\mathrm{BnONa}$ yielded hydroxylated ester $( \pm)-\mathbf{8}$. Again, the hydroxyl group was attached to the $\mathrm{C}-5$ ring atom with the ester and carbamate moieties in a trans relative relationship. After a few experimental attempts, fluorination of $( \pm)-8$ took place successfully with 3 equiv of Deooxfluor. The highest yield (65\%) was attained in $\mathrm{CH}_{2} \mathrm{Cl}_{2}$ at room temperature when fluorinated product $( \pm)-9$ formed through inversion was isolated by column chromatography (Scheme 2 ). 
<smiles>CC(=O)NC1C[C@H](I)[C@@H]2CC(=O)O[C@H]1C2=O</smiles>

(士) 5
(士) 6

DBU, THF

$65{ }^{\mathrm{s}} \mathrm{C}, 6 \mathrm{~h}, 80 \%$<smiles>O=C(OCc1ccccc1)OC(=O)[C@@H]1CC(F)C=CC1OCc1ccccc1</smiles>

(士) -9

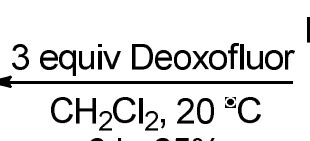

$3 \mathrm{~h}, 65 \%$

$\mathrm{HO}$

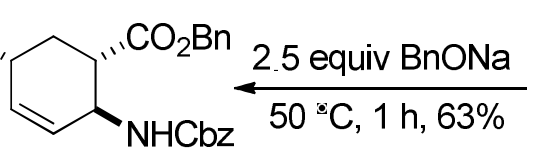

(士) 8

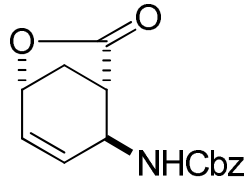

(士)-7

Scheme 2.

Next, we extended our study by changing the position of the hydroxyl group in the benzyl/benzyloxycarbonyl protected amino acid. Accordingly, Cbz-protected amino acid ( \pm )-10 (derived from ( \pm )-1) [6] was first transformed into the corresponding benzyl ester $( \pm)-11$ which, in turn, led to all-cis epoxide ( \pm )-12 by stereoselective oxirane formation with $m$-chloroperbenzoic acid (MCPBA), which involved the hydrogen bonding directing effect. Analogously to our earlier findings [7], oxirane opening using slightly modified reductive opening methods furnished regioselectively all-cis hydroxylated amino ester $( \pm)-\mathbf{1 3}$, which contains the hydroxyl function at C-4 of the ring.<smiles>NC1CC=CCC1C(=O)[O-]</smiles>

(士)-1<smiles>O=C(O)C1CC=CCC1NC=[18O]</smiles>

$( \pm)-10$

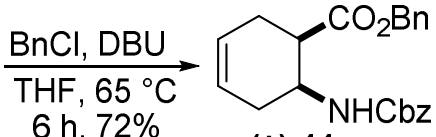

(士)-11

$6 \mathrm{~h}, 72 \%$

MCPBA, $\mathrm{CH}_{2} \mathrm{Cl}_{2}$

$20^{\circ} \mathrm{C}, 12 \mathrm{~h}, 61 \%$<smiles>CC(=O)NC1C=C[C@H](CBr)CCC1C(=O)NC1CC=CCC1C(=O)OCc1ccccc1</smiles>

(士)-11
(士)-14 $\frac{\begin{array}{c}1.5 \text { equiv } \\ \text { Deoxofluor }\end{array}}{\mathrm{CH}_{2} \mathrm{Cl}_{2}}$

$0{ }^{\circ} \mathrm{C}, 3 \mathrm{~h}$
$72 \%$<smiles>O=C(NC1CC(O)CCC1C(=O)OCc1ccccc1)Oc1ccccc1</smiles>

(士)-13

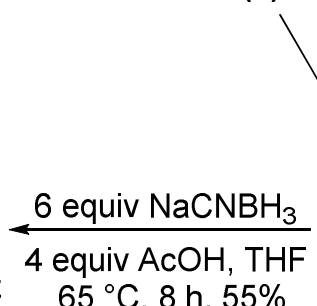

$65^{\circ} \mathrm{C}, 8 \mathrm{~h}, 55 \%$ 1.2:1

\section{Scheme 3.}


Hydroxylated cyclohexane amino ester $( \pm)$-13 was subjected to fluorination under various experimental conditions (temperature, solvent, equivalents of fluorinating agent). Unfortunately, in all cases only a mixture of unseparable elimination products $( \pm)-11$ and $( \pm)-14$ in approximately 1.2:1 ratio could be isolated (Scheme 3). Note that the elimination products alongside substitution derivatives are often formed in nucleophilic fluorination reactions.

Next, our intention was to evaluate the behavior under fluorination of a trans cyclohexane amino ester. Amino acid ( \pm )-15 (derived from ( \pm )-5) [6] provided the corresponding trans amino ester $( \pm)-\mathbf{1 6}$ by benzylation under basic conditions. Epoxidation of this product was accomplished stereoselectively with MCPBA to afford oxirane derivative $( \pm)-17$. trans Epoxide ( \pm )-17, contrary to its cis counterpart $( \pm)-\mathbf{1 2}$, under modified reductive heteroring opening conditions gave regioselectively hydroxylated amino ester $( \pm)-18$, with the hydroxy group located at C-5 (for an earlier analogous transformation, see ref. 7). Compound ( \pm )-18 was then submitted to fluorination under various conditions. The reaction, when carried out with Deoxofluor at $20{ }^{\circ} \mathrm{C}$, yielded only compound ( \pm )-16 formed as a result of elimination. In sharp contrast, a similar fluorination performed at $0{ }^{\circ} \mathrm{C}$ gave fluorinated derivative $( \pm)-19$ as the sole product in moderate yield by substitution with inversion (Scheme 4).

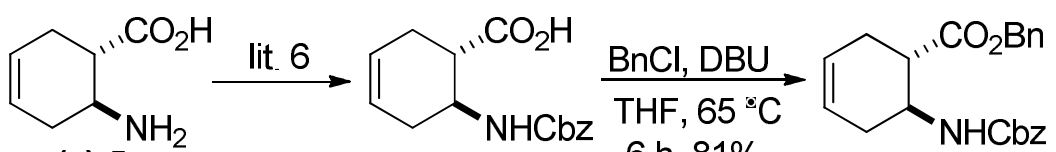

$( \pm)-5$

$( \pm)-15$

$6 \mathrm{~h}, 81 \%$

$( \pm)-16$

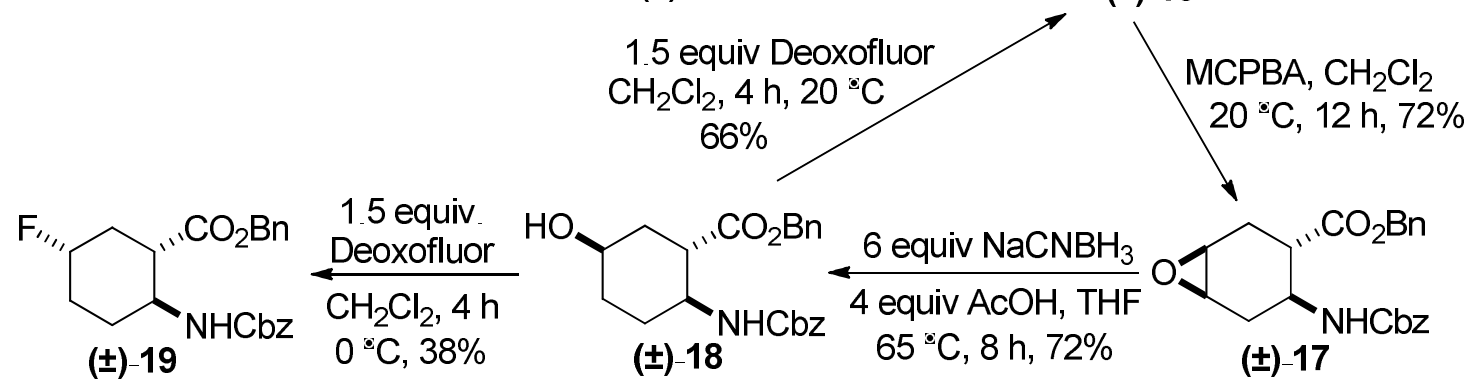

Scheme 4.

In conclusion, the fluorination of various hydroxylated cyclohexene or cyclohexane $\beta$ protected amino ester regio- and stereoisomers has been investigated under varied experimental conditions. It was found that both the stereochemistry of the starting substances and the position of 
the hydroxyl group have essential roles on the outcome of fluorination. Further investigations with other novel substrates will also be studied in our laboratory.

\section{Acknowledgments}

We are grateful to the Hungarian Research Foundation (NKFIH Nos. K 115731 and K 119282) for financial support. The financial support of the GINOP-2.3.2-15-2016-00038 project is also acknowledged. This research was supported by the EU-funded Hungarian grant EFOP-3.6.1-162016-00008.

\section{Experimental}

Benzyl $\left(\left(1 R^{*}, 2 S^{*}, 5 S^{*}\right)-7-0 x 0-6-0 x a b i c y c l o[3.2 .1]\right.$ oct-3-en-2-yl)carbamate, $( \pm)-3$<smiles>O=C(N[C@H]1C=C[C@H]2C[C@H]1C(=O)O2)OCc1ccccc1</smiles>

To a solution of iodolactone $( \pm)-2(20.5 \mathrm{mmol})$ in THF $(50 \mathrm{~mL})$, DBU $(45.1 \mathrm{mmol})$ was added and the reaction mixture was stirred under reflux for $6 \mathrm{~h}$. It was then diluted with EtOAc $(50 \mathrm{~mL})$, washed with $\mathrm{H}_{2} \mathrm{O}(3 \times 30 \mathrm{~mL})$, dried $\left(\mathrm{Na}_{2} \mathrm{SO}_{4}\right)$ and concentrated under reduced pressure. The crude product was then purified by column chromatography on silica gel ( $n$-hexane/EtOAc). A white solid; yield: 75\%; $\mathrm{R}_{\mathrm{f}}=0.3$ ( $n$-hexane/EtOAc 2:1); Mp 102-104 ${ }^{\circ} \mathrm{C} ;{ }^{1} \mathrm{H}$ NMR $(400 \mathrm{MHz}$, $\left.\mathrm{CDCl}_{3}\right): \delta=2.17-2.24\left(\mathrm{~m}, 1 \mathrm{H}, \mathrm{CH}_{2}\right), 2.56-2.65\left(\mathrm{~m}, 1 \mathrm{H}, \mathrm{CH}_{2}\right), 3.04-3.09$ (m, 1H, H-1), 4.77 (brs, 1H, N-H), 4.87-4.91 (m, 1H, H-2), 5.06-5.23 (m, 3H, H-5, $\left.\mathrm{OCH}_{2}\right)$, 5.77-5.84 (m, 1H, H-3), 6.33$6.40(\mathrm{~m}, 1 \mathrm{H}, \mathrm{H}-4), 7.32-7.41$ (m, 5H, CH-Ar); $\left.{ }^{13} \mathrm{C} \mathrm{NMR} \mathrm{(100} \mathrm{MHz,} \mathrm{CDCl}_{3}\right): \delta=36.6,43.6,49.0$, 67.1, 73.0, 128.1. 128.3, 128.5, 131.1, 131.2, 133.8, 155.4, 176.1; MS (ESI, pos) $\mathrm{m} / \mathrm{z}=547$ $[2 \mathrm{M}+1]$; Anal. Calcd. for $\mathrm{C}_{15} \mathrm{H}_{15} \mathrm{NO}_{4}: \mathrm{C}, 65.92 ; \mathrm{H}, 5.53 ; \mathrm{N}, 5.13$. Found: C, 65.59; H, 5.16; N, 4.79 .

Benzyl $\quad\left(1 R^{*}, 2 S^{*}, 5 S^{*}\right)-2-((($ benzyloxy$)$ carbonyl)amino)-5-hydroxycyclohex-3-enecarboxylate, $( \pm)-4$ 


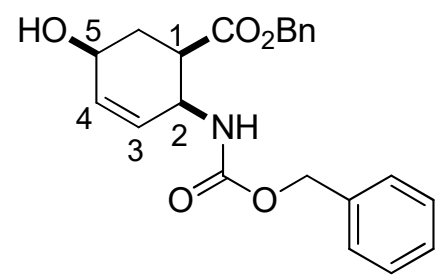

To unsaturated lactone $( \pm)-3(14.6 \mathrm{mmol}) \mathrm{BnONa}(35.1 \mathrm{mmol})$ was added and the mixture was stirred for $6 \mathrm{~h}$ at $50{ }^{\circ} \mathrm{C}$. Then the reaction mixture was diluted with EtOAc $(75 \mathrm{~mL})$, washed with $\mathrm{H}_{2} \mathrm{O}(3 \times 30 \mathrm{~mL})$, dried $\left(\mathrm{Na}_{2} \mathrm{SO}_{4}\right)$ and concentrated under reduced pressure. The crude reaction mixture was then purified by column chromatography on silica gel ( $n$-hexane/EtOAc).

A white solid; yield: 55\%; $\mathrm{R}_{\mathrm{f}}=0.5$ (n-hexane/EtOAc 1:1); Mp 48-50 ${ }^{\circ} \mathrm{C} ;{ }^{1} \mathrm{H}$ NMR $(400 \mathrm{MHz}$, $\left.\mathrm{CDCl}_{3}\right): \delta=2.00-2.08\left(\mathrm{~m}, 2 \mathrm{H}, \mathrm{CH}_{2}\right), 2.89-3.00(\mathrm{~m}, 1 \mathrm{H}, \mathrm{H}-1), 4.22($ brs, $1 \mathrm{H}, \mathrm{N}-\mathrm{H}), 4.43(\mathrm{~m}, 1 \mathrm{H}, \mathrm{H}-$ 2), 4.90-5.16 (m, 5H, H-5 and $\left.\mathrm{OCH}_{2}\right), 5.70-5.79$ (m, 1H, H-3), 5.84-5.95 (m, 1H, H-4), 7.27-7.37 (m, 10H, CH-Ar); ${ }^{13} \mathrm{C}$ NMR (100 MHz, $\left.\mathrm{CDCl}_{3}\right): \delta=14.1,32.3,42.9,49.8,61.5,62.1,66,4,127.5$, 127.8, 128.6, 129.1, 131.0, 131.2, 132.3, 136.2, 137.0, 155.6, 173.4; MS (ESI, pos) $\mathrm{m} / \mathrm{z}=404$ $[\mathrm{M}+\mathrm{Na}]$; Anal. Calcd. for $\mathrm{C}_{22} \mathrm{H}_{23} \mathrm{NO}_{5}: \mathrm{C}, 69.28 ; \mathrm{H}, 6.08 ; \mathrm{N}, 3.67$. Found: C, 69.55; H, 5.76; N, 3.32 .

\section{Benzyl $\left(\left(1 S^{*}, 2 S^{*}, 5 R^{*}\right)-7-0 x 0-6-0 x a b i c y c l o[3.2 .1]\right.$ oct-3-en-2-yl)carbamate, $( \pm)-7$}<smiles>O=C(NC1C=CC2C[C@H]1C(=O)O2)OCc1ccccc1</smiles>

To a solution of iodolactone $( \pm)-6(20.51 \mathrm{mmol})$ in THF $(50 \mathrm{~mL})$ DBU $(45.12 \mathrm{mmol})$ was added and the reaction mixture was stirred under reflux for $6 \mathrm{~h}$. It was then diluted with EtOAc $(50 \mathrm{~mL})$, washed with $\mathrm{H}_{2} \mathrm{O}(3 \times 30 \mathrm{~mL})$, dried $\left(\mathrm{Na}_{2} \mathrm{SO}_{4}\right)$ and concentrated under reduced pressure. The crude reaction mixture was then purified by column chromatography on silica gel ( $n$ hexane/EtOAc).

A white solid; yield: 80\%; $\mathrm{R}_{\mathrm{f}}=0.3$ ( $n$-hexane/EtOAc2:1 ); Mp 125-127 ${ }^{\circ} \mathrm{C} ;{ }^{1} \mathrm{H}$ NMR $(400 \mathrm{MHz}$, $\left.\mathrm{CDCl}_{3}\right): \delta=1.59-1.92\left(\mathrm{~m}, 1 \mathrm{H}, \mathrm{CH}_{2}\right), 2.11-2.44\left(\mathrm{~m}, 2 \mathrm{H}, \mathrm{H}-1\right.$ and $\left.\mathrm{CH}_{2}\right), 3.02-3.22(\mathrm{~m}, 1 \mathrm{H}, \mathrm{H}-2)$, 4.53 (brs, 1H, N-H), 4.79 (m, 1H, H-5), 4.94-5.21 (m, 2H, $\left.\mathrm{OCH}_{2}\right)$, 5.70-5.76 (m, 1H, H-3), 6.366.43 (m, 1H, H-4), 7.26-7.39 (m, 5H, CH-Ar); $\left.{ }^{13} \mathrm{C} \mathrm{NMR} \mathrm{(100} \mathrm{MHz,} \mathrm{CDCl}_{3}\right): \delta=31.5,43.4,47.3$, 
$67.2,72.9,128.3,128.4,128.6,133.2,133.3,135.6,155.2,176.6$; MS (ESI, pos) $\mathrm{m} / \mathrm{z}=547$ [2M+1]; Anal. Calcd. for $\mathrm{C}_{15} \mathrm{H}_{15} \mathrm{NO}_{4}: \mathrm{C}, 65.92 ; \mathrm{H}, 5.53 ; \mathrm{N}, 5.13$. Found: $\mathrm{C}, 65.60 ; \mathrm{H}, 5.18$; N, 5.46 .

\section{Benzyl}

$\left(1 S^{*}, 2 S^{*}, 5 R^{*}\right)-2-(((b e n z y l o x y)$ carbonyl)amino)-5-hydroxycyclohex-3-enecarboxylate, $( \pm)-8$

HO,<smiles>O=C(NC1C=CCCC1C(=O)OCc1ccccc1)OCc1ccccc1</smiles>

To unsaturated lactone $( \pm)-7(14.6 \mathrm{mmol}) \mathrm{BnONa}(35.1 \mathrm{mmol})$ was added and the solution was stirred for $6 \mathrm{~h}$ at $50{ }^{\circ} \mathrm{C}$. The reaction mixture was then diluted with EtOAc $(75 \mathrm{~mL})$, washed with $\mathrm{H}_{2} \mathrm{O}(3 \times 30 \mathrm{~mL})$, dried $\left(\mathrm{Na}_{2} \mathrm{SO}_{4}\right)$ and concentrated under reduced pressure.

A white solid; yield: 63\%; $\mathrm{R}_{\mathrm{f}}=0.3$ ( $n$-hexane/EtOAc 1:1); Mp 138-140 ${ }^{\circ} \mathrm{C} ;{ }^{1} \mathrm{H}$ NMR $(400 \mathrm{MHz}$, $\left.\mathrm{CDCl}_{3}\right): \delta=1.86-1.98\left(\mathrm{~m}, 1 \mathrm{H}, \mathrm{CH}_{2}\right), 2.02-2.11\left(\mathrm{~m}, 1 \mathrm{H}, \mathrm{CH}_{2}\right), 2.27-2.36(\mathrm{~m}, 1 \mathrm{H}, \mathrm{H}-1), 2.74-2.85$ (m, 1H, H-2), 4.32 (brs, 1H, N-H), 4.51-4.63 (m, 1H, H-5), 4.82 (brs, 1H, O-H), 5.02-5.21 (m, $4 \mathrm{H}, \mathrm{OCH}_{2}$ ), 5.67-5.75 (m, 1H, H-3), 5.85-5.92 (m, 1H, H-4), 7.32-7.41 (m, 10H, CH-Ar); ${ }^{13} \mathrm{C}$ NMR (100 MHz, $\left.\mathrm{CDCl}_{3}\right): \delta=33.8,45.6,49.5,54.3,60.6,65.6,77.1,77.7,128.5,128.6,128.7$, 128.9, 129.1, 133.5, 136.6, 155.9, 173.4; MS (ESI, pos) m/z = $382[\mathrm{M}+1]$; Anal. Calcd. for $\mathrm{C}_{22} \mathrm{H}_{23} \mathrm{NO}_{5}$ : C, 69.28; H, 6.08; N, 3.67. Found: C, 67.89; H, 5.77; N, 3.99.

\section{Benzyl $\left(1 S^{*}, 2 S^{*}, 5 S^{*}\right)-2-(((b e n z y l o x y)$ carbonyl)amino)-5-fluorocyclohex-3-enecarboxylate,} $( \pm)-9$<smiles>O=C(NC1C=CC(F)C[C@H]1C(=O)O)OCc1ccccc1</smiles>

To a solution of hydroxylated ester $( \pm)-8(0.52 \mathrm{mmol})$ in dry $\mathrm{CH}_{2} \mathrm{Cl}_{2}(5 \mathrm{~mL})$ Deoxofluor solution (50\% in toluene, $1.56 \mathrm{mmol}$ ) was added under argon. The reaction mixture was stirred at room temperature for $3 \mathrm{~h}$, and then diluted with $\mathrm{CH}_{2} \mathrm{Cl}_{2}(20 \mathrm{~mL})$, washed with saturated $\mathrm{NaHCO}_{3}(3 \mathrm{x} 10$ 
$\mathrm{mL})$ and $\mathrm{H}_{2} \mathrm{O}(2 \times 10 \mathrm{~mL})$, dried $\left(\mathrm{Na}_{2} \mathrm{SO}_{4}\right)$ and concentrated under reduced pressure. The crude residue was purified by column chromatography on silica gel ( $n$-hexane/EtOAc).

A white solid; yield: $65 \%$; $\mathrm{R}_{\mathrm{f}}=0.3$ (n-hexane/EtOAc 3:1); Mp 63-65 ${ }^{\circ} \mathrm{C} ;{ }^{1} \mathrm{H}$ NMR $(400 \mathrm{MHz}$, $\left.\mathrm{CDCl}_{3}\right): \delta=2.01-2.22\left(\mathrm{~m}, 1 \mathrm{H}, \mathrm{CH}_{2}\right), 2.27-2.39\left(\mathrm{~m}, 1 \mathrm{H}, \mathrm{CH}_{2}\right), 2.81-2.90(\mathrm{~m}, 1 \mathrm{H}, \mathrm{H}-1), 4.48-4.62$ (m, 1H, H-2), 4.82-4.98 (m, 1H, H-5), 5.01-5.21 (m, 4H, $\mathrm{OCH}_{2}$ ), 5.22-5.36 (brs, 1H, N-H), 5.955.99 (m, 2H, H-3 and H-4), 7.32-7.42 (m, 10H, CH-Ar); ${ }^{13} \mathrm{C}$ NMR (100 MHz, $\left.\mathrm{CDCl}_{3}\right): \delta=30.6$ (d, $J=26.8), 41.3,42.2,50.0,67.3,81.5$ (d, $J=170.8), 125.9,126.0,128.5,128.9,135.3,135.4$, 136.6, 156.0, 173.0; ${ }^{19} \mathrm{~F}$ NMR (100 MHz, $\left.\mathrm{CDCl}_{3}\right): \delta=-165.7$; MS (ESI, pos) $\mathrm{m} / \mathrm{z}=406[\mathrm{M}+\mathrm{Na}]$; Anal. Calcd. for $\mathrm{C}_{22} \mathrm{H}_{22} \mathrm{FNO}_{4}$ : C, 68.92; H, 5.78; N, 3.65. Found: C, 68.62; H, 5.41; N, 3.92 .

\section{Benzyl $\left(1 R^{*}, 6 S^{*}\right)-6-((($ benzyloxy)carbonyl)amino)cyclohex-3-enecarboxylate, $( \pm)-11$}<smiles>O=C(NC1CC=CCC1C(=O)Oc1ccccc1)OCc1ccccc1</smiles>

To a solution of amino acid ( \pm -10 (28.4 mmol) in THF (75 mL), DBU (42.6 mmol) and BnBr $(39.8 \mathrm{mmol})$ were added and the reaction mixture was stirred under reflux for $6 \mathrm{~h}$. The reaction mixture was diluted with EtOAc $(50 \mathrm{~mL})$, washed with $\mathrm{H}_{2} \mathrm{O}(3 \times 30 \mathrm{~mL})$, dried $\left(\mathrm{Na}_{2} \mathrm{SO}_{4}\right)$ and concentrated under reduced pressure.

A white solid; yield: 72\%; $\mathrm{R}_{\mathrm{f}}=0.6$ (n-hexane/EtOAc 3:1); Mp $42-43{ }^{\circ} \mathrm{C} ;{ }^{1} \mathrm{H}$ NMR $(400 \mathrm{MHz}$, $\left.\mathrm{CDCl}_{3}\right): \delta=2.12-2.46\left(\mathrm{~m}, 4 \mathrm{H}, \mathrm{CH}_{2}\right), 2.49-2.61$ (m, 1H, H-1), 2.84-2.91 (m, 1H, H-2), 4.29 (brs, 1H, N-H), 5.02-5.16 (m, 4H, $\left.\mathrm{OCH}_{2}\right)$, 5.57-5.70 (m, 2H, H-4, H-5), 7.26-7.39 (m, 10H, CH-Ar); ${ }^{13} \mathrm{C}$ NMR (100 MHz, $\left.\mathrm{CDCl}_{3}\right): \delta=24.9,31.0,42.1,46.8,66.6$. 66.7. 124.1, 124.7, 124.9, 128.1, 128.2, 128.4, 128.5, 136.4, 136.5, 155.5, 173.2; MS (ESI, pos) m/z = 366 [M+1]; Anal. Calcd. for $\mathrm{C}_{22} \mathrm{H}_{23} \mathrm{NO}_{4}$ : C, 72.31; H, 6.34; N, 3.83. Found: C, 72.66; H, 6.01; N, 3.50.

Benzyl $\quad\left(1 S^{*}, 3 R^{*}, 4 S^{*}, 6 R^{*}\right)-4-((($ benzyloxy)carbonyl)amino)-7-oxabicyclo[4.1.0]heptane-3carboxylate, $( \pm)-12$ 
<smiles>O=C(NC1CC2OC2CC1C(=O)OCc1ccccc1)OCc1ccccc1</smiles>

To a solution of amino ester $( \pm)-11(13.3 \mathrm{mmol})$ in $\mathrm{CH}_{2} \mathrm{Cl}_{2}(60 \mathrm{~mL}) \mathrm{MCPBA}(13.3 \mathrm{mmol})$ was added at $0{ }^{\circ} \mathrm{C}$. The reaction mixture was stirred at room temperature for $12 \mathrm{~h}$, then diluted with $\mathrm{CH}_{2} \mathrm{Cl}_{2}(50 \mathrm{~mL})$, washed with $\mathrm{H}_{2} \mathrm{O}(3 \times 30 \mathrm{~mL})$, dried $\left(\mathrm{Na}_{2} \mathrm{SO}_{4}\right)$ and concentrated under reduced pressure. The crude residue was purified by column chromatography on silica gel ( $n$ hexane/EtOAc).

A white solid; yield: $61 \%$; $\mathrm{R}_{\mathrm{f}}=0.5$ (n-hexane/EtOAc 2:1); Mp 53-56 ${ }^{\circ} \mathrm{C}$; ${ }^{1} \mathrm{H}$ NMR $(400 \mathrm{MHz}$, $\left.\mathrm{CDCl}_{3}\right): \delta=2.06-2.32\left(\mathrm{~m}, 3 \mathrm{H}, \mathrm{CH}_{2}\right), 2.54-2.61\left(\mathrm{~m}, 1 \mathrm{H}, \mathrm{CH}_{2}\right), 2.64-2.73(\mathrm{~m}, 1 \mathrm{H}, \mathrm{H}-3), 3.20-3.26$ (m, 2H, H-1 and H-6), 4.12-4.24 (m, 1H, H-4), 5.04-5.19 (m, 4H, $\left.\mathrm{OCH}_{2}\right), 5.77-5.85$ (brs, $1 \mathrm{H}, \mathrm{N}-$ H), 7.31-7.44 (m, 10H, CH-Ar); ${ }^{13} \mathrm{C}$ NMR (100 MHz, $\left.\mathrm{CDCl}_{3}\right): \delta=24.4,29.4,40.7,46.0,50.9$, 51.6, 66.8, 66.9, 127.1, 128.0, 128.2, 128.5, 128.6, 135.7, 136.5, 155.7, 172.6; MS (ESI, pos) m/z $=382[\mathrm{M}+1]$; Anal. Calcd. for $\mathrm{C}_{22} \mathrm{H}_{23} \mathrm{NO}_{5}: \mathrm{C}, 69.28 ; \mathrm{H}, 6.08 ; \mathrm{N}, 3.67$. Found: C, 68.87; H, 5.80; $\mathrm{N}, 3.40$.

\section{Benzyl $\left(1 R^{*}, 2 S^{*}, 4 S^{*}\right)-2-((($ benzyloxy)carbonyl)amino)-4-hydroxycyclohexanecarboxylate, $( \pm)-13$}<smiles>O=C(NC1CC(O)CCC1C(=O)OCc1ccccc1)OCc1ccccc1</smiles>

To a solution of epoxy amino ester $( \pm)-12(7.8 \mathrm{mmol})$ in $\mathrm{THF}, \mathrm{NaCNBH}_{3}(62.9 \mathrm{mmol})$ and $\mathrm{CH}_{3} \mathrm{COOH}$ (47.2 mmol) were added. The reaction mixture was stirred for $8 \mathrm{~h}$ under reflux, then diluted with EtOAc, washed with $\mathrm{H}_{2} \mathrm{O}$, dried $\left(\mathrm{Na}_{2} \mathrm{SO}_{4}\right)$ and concentrated under reduced pressure. The crude product was purified by column chromatography on silica gel ( $n$-hexane/EtOAc). A white solid; yield: 55\%; $\mathrm{R}_{\mathrm{f}}=0.3$ (n-hexane/EtOAc 1:1); Mp 70-72 ${ }^{\circ} \mathrm{C} ;{ }^{1} \mathrm{H}$ NMR $(400 \mathrm{MHz}$, $\left.\mathrm{CDCl}_{3}\right): \delta=1.73-2.00\left(\mathrm{~m}, 6 \mathrm{H}, \mathrm{CH}_{2}\right), 2.10-2.22$ (m, 1H, H-1), 2.68-2.76 (m, 1H, H-2), 4.07 (brs, 1H, H-4), 4.33 (m, 1H, O-H), 5.00-5.14 (m, 4H, OCH 2 ), 6.40-6.52 (brs, 1H, N-H), 7.30-7.40 (m, $10 \mathrm{H}, \mathrm{CH}-\mathrm{Ar}) ;{ }^{13} \mathrm{C} \mathrm{NMR}\left(100 \mathrm{MHz}, \mathrm{CDCl}_{3}\right): \delta=31.3,37.4,44.4,48.2,66.5,65.6,66.7,128.0$, 
128.2, 128.3, 128.5, 135.6, 136.4, 155.7, 173.1; MS (ESI, pos) $\mathrm{m} / \mathrm{z}=382$ [M+1]; Anal. Calcd. for $\mathrm{C}_{22} \mathrm{H}_{25} \mathrm{NO}_{5}$ : C, 68.91; H, 6.57; N, 3.65. Found: C, 68.56; H, 6.19; N, 3.31.

\section{Benzyl $\left(1 S^{*}, 6 S^{*}\right)-6-((($ benzyloxy)carbonyl)amino)cyclohex-3-enecarboxylate, $( \pm)-16$<smiles>O=C(NC1CC=CCC1C(=O)Oc1ccccc1)OCc1ccccc1</smiles>

To a solution of amino acid $( \pm)-15(29.0 \mathrm{mmol})$ in THF $(75 \mathrm{~mL})$ DBU $(43.6 \mathrm{mmol})$ and $\mathrm{BnBr}(40.7$ mmol) were added and the reaction mixture was stirred under reflux for $6 \mathrm{~h}$. The reaction mixture was diluted with EtOAc $(50 \mathrm{~mL})$, washed with $\mathrm{H}_{2} \mathrm{O}(3 \times 30 \mathrm{~mL})$, dried $\left(\mathrm{Na}_{2} \mathrm{SO}_{4}\right)$ and concentrated under reduced pressure. The residue was purified by column chromatography on silica gel ( $n$ hexane/EtOAc).

A white solid; yield: 81\%; $\mathrm{R}_{\mathrm{f}}=0.5$ (n-hexane/EtOAc 3:1); Mp 45-46 ${ }^{\circ} \mathrm{C} ;{ }^{1} \mathrm{H}$ NMR $(400 \mathrm{MHz}$, $\left.\mathrm{CDCl}_{3}\right): \delta=1.95-2.06\left(\mathrm{~m}, 1 \mathrm{H}, \mathrm{CH}_{2}\right), 2.30-2.40(\mathrm{~m}, 1 \mathrm{H} \mathrm{CH} 2), 2.47-2.62\left(\mathrm{~m}, 2 \mathrm{H}, \mathrm{CH}_{2}\right), 2.76-2.85$ (m, 1H, H-1), 4.11-4.22 (m, 1H, H-2), 4.88 (brs, 1H, N-H), 5.04-5.17 (m, 4H, OCH $)$, 5.59-5.73 (m, 2H, H-4, H-5), 7.30-7.42 (m, 10H, CH-Ar); ${ }^{13} \mathrm{C} \mathrm{NMR} \mathrm{(100} \mathrm{MHz,} \mathrm{CDCl}_{3}$ ): $\delta=26.7,31.1,44.5$, $47.9,66.5,66.6,124.2,124.9,128.4,128.5,135.8,136.4,155.5,172.3 ;$ MS (ESI, pos) $\mathrm{m} / \mathrm{z}=366$ $[\mathrm{M}+1]$; Anal. Calcd. for $\mathrm{C}_{22} \mathrm{H}_{23} \mathrm{NO}_{4}$ : C, 72.31; H, 6.34; N, 3.83. Found: C, 72.68; H, 6.70; N, 3.51.

\section{Benzyl $\quad\left(1 S^{*}, 3 S^{*}, 4 S^{*}, 6 R^{*}\right)-4-((($ benzyloxy)carbonyl)amino)-7-oxabicyclo[4.1.0]heptane-3-} carboxylate, $( \pm)-17$<smiles>O=C(NC1CC2OC2C1)OCc1ccccc1</smiles>

To a solution of amino ester $( \pm)-16(17.0 \mathrm{mmol})$ in $\mathrm{CH}_{2} \mathrm{Cl}_{2}(60 \mathrm{~mL}) \mathrm{MCPBA}(17.0 \mathrm{mmol})$ was added at $0{ }^{\circ} \mathrm{C}$. The reaction mixture was stirred at room temperature for $12 \mathrm{~h}$, then diluted with $\mathrm{CH}_{2} \mathrm{Cl}_{2}(50 \mathrm{~mL})$, washed with $\mathrm{H}_{2} \mathrm{O}(3 \times 30 \mathrm{~mL})$, dried $\left(\mathrm{Na}_{2} \mathrm{SO}_{4}\right)$ and concentrated under reduced pressure. The crude residue was purified by column chromatography on silica gel (nhexane/EtOAc). 
A white solid; yield: 72\%; $\mathrm{R}_{\mathrm{f}}=0.5$ (n-hexane/EtOAc 2:1); Mp 89-91 ${ }^{\circ} \mathrm{C} ;{ }^{1} \mathrm{H}$ NMR $(400 \mathrm{MHz}$, $\left.\mathrm{CDCl}_{3}\right): \delta=1.90-1.98\left(\mathrm{~m}, 1 \mathrm{H}, \mathrm{CH}_{2}\right), 2.14-2.31\left(\mathrm{~m}, 2 \mathrm{H}, \mathrm{CH}_{2}\right), 2.35-2.45\left(\mathrm{~m}, 1 \mathrm{H}, \mathrm{CH}_{2}\right), 2.73-2.81$ (m, 1H, H-3), 3.16-3.21 (m, 1H, H-4), 3.27-3.32 (m, 1H, H-1), 4.07-4.20 (m, 1H, H-6), 5.03-5.20 $\left(\mathrm{m}, 4 \mathrm{H}, \mathrm{OCH}_{2}\right), 5.36-5.44(\mathrm{~m}, 1 \mathrm{H}, \mathrm{N}-\mathrm{H}), 7.30-7.42(\mathrm{~m}, 10 \mathrm{H}, \mathrm{CH}-\mathrm{Ar}) ;{ }^{13} \mathrm{C} \mathrm{NMR}(100 \mathrm{MHz}$, $\left.\mathrm{CDCl}_{3}\right): \delta=23.8,28.4,41.1,46.5,51.6,52.4,66.7,66.8,128.5,128.6,128.7,128.8,135.6,136.4$, $155.4,172.8$

MS (ESI, pos) $\mathrm{m} / \mathrm{z}=382[\mathrm{M}+1]$; Anal. Calcd. for $\mathrm{C}_{22} \mathrm{H}_{23} \mathrm{NO}_{5}$ : C, 69.28; H, 6.08; N, 3.67. Found: C, 68.88; H, 5.79; N, 3.38.

\section{Benzyl $\left(1 S^{*}, 2 S^{*}, 5 R^{*}\right)-2-(((b e n z y l o x y)$ carbonyl)amino)-5-hydroxycyclohexanecarboxylate, $( \pm)-18$}<smiles>O=C(NC1CCC(O)C[C@H]1C(=O)OCc1ccccc1)OCc1ccccc1</smiles>

To a solution of compound $( \pm)-17$ epoxy amino ester $(10.4 \mathrm{mmol})$ in $\mathrm{THF} \mathrm{NaCNBH}_{3}(31.4 \mathrm{mmol})$ and $\mathrm{CH}_{3} \mathrm{COOH}(10.4 \mathrm{mmol})$ were added. The reaction mixture was stirred for $8 \mathrm{~h}$ under reflux, than diluted with EtOAc, washed with $\mathrm{H}_{2} \mathrm{O}(3 \times 30 \mathrm{~mL})$, dried $\left(\mathrm{Na}_{2} \mathrm{SO}_{4}\right)$ and concentrated under reduced pressure. The crude product was purified by column chromatography on silica gel ( $n$ hexane/EtOAc).

A white solid; yield: 72\%; $\mathrm{R}_{\mathrm{f}}=0.6$ (n-hexane/EtOAc 1:1); Mp 58-60 ${ }^{\circ} \mathrm{C}$; ${ }^{1} \mathrm{H}$ NMR $(400 \mathrm{MHz}$, $\left.\mathrm{CDCl}_{3}\right): \delta=1.52-2.13\left(\mathrm{~m}, 7 \mathrm{H}, \mathrm{CH}_{2}, \mathrm{H}-1\right), 2.76-2.86(\mathrm{~m}, 1 \mathrm{H}, \mathrm{H}-2), 3.77-3.88(\mathrm{~m}, 1 \mathrm{H}, \mathrm{H}-5), 4.08$ (brs, 1H, N-H), 4.78 (brs, 1H, O-H), 4.98-5.10 (m, 4H, $\left.\mathrm{OCH}_{2}\right), 7.26-7.37$ (m, 10H, CH-Ar); ${ }^{13} \mathrm{C}$ NMR (100 MHz, $\left.\mathrm{CDCl}_{3}\right): \delta=26.6,29.7,34.7,43.9,51.1,64.4,66.6,66.7,128.0,128.1,128.4$, 128.5, 135.8, 136.5, 155.5, 173.8; MS (ESI, pos) $\mathrm{m} / \mathrm{z}=384$ [M+1]; Anal. Calcd. for $\mathrm{C}_{22} \mathrm{H}_{25} \mathrm{NO}_{5}$ : C, 68.91; H, 6.57; N, 3.65. Found: C, 68.56; H, 6.21; N, 3.99.

Benzyl $\left(1 S^{*}, 2 S^{*}, 5 S^{*}\right)-2-((($ benzyloxy$)$ carbonyl)amino)-5-fluorocyclohexanecarboxylate, $( \pm)-$ 19<smiles>O=C(NC1CCC(F)C[C@@H]1C(=O)OCc1ccccc1)OCc1ccccc1</smiles> 
To a solution of hydroxylated ester $( \pm)-\mathbf{1 8}(0.52 \mathrm{mmol})$ in dry $\mathrm{CH}_{2} \mathrm{Cl}_{2}(5 \mathrm{~mL})$ Deoxofluor solution $(50 \%$ in toluene, $1.56 \mathrm{mmol})$ was added under an argon. The reaction mixture was stirred at $0{ }^{\circ} \mathrm{C}$ for $4 \mathrm{~h}$, and then diluted with $\mathrm{CH}_{2} \mathrm{Cl}_{2}(20 \mathrm{~mL})$, washed with saturated $\mathrm{NaHCO}_{3}(3 \times 15 \mathrm{~mL})$ and then $\mathrm{H}_{2} \mathrm{O}(2 \times 10 \mathrm{~mL})$, dried $\left(\mathrm{Na}_{2} \mathrm{SO}_{4}\right)$ and concentrated under reduced pressure. The crude product was purified by column chromatography on silica gel ( $n$-hexane/EtOAc).

A white solid; yield: $38 \%$; $\mathrm{R}_{\mathrm{f}}=0.6$ (n-hexane/EtOAc 3:1); Mp 77-79 ${ }^{\circ} \mathrm{C} ;{ }^{1} \mathrm{H}$ NMR $(400 \mathrm{MHz}$, $\left.\mathrm{CDCl}_{3}\right): \delta=1.41-1.49\left(\mathrm{~m}, 1 \mathrm{H}, \mathrm{CH}_{2}\right), 1.62-1.71\left(\mathrm{~m}, 1 \mathrm{H}, \mathrm{CH}_{2}\right), 1.87-1.96\left(\mathrm{~m}, 1 \mathrm{H}, \mathrm{CH}_{2}\right), 2.03-2.10$ (m, 2H, $\mathrm{CH}_{2}$ ), 1.28-1.35 (m, 1H, $\mathrm{CH}_{2}$ ), 2.44-2.52 (m, 1H, H-1), 3.60-3.67 (m, 1H, H-2), 4.41-4.60 (m, 1H, H-5), 4.78 (brs, 1H, N-H), 5.03-5.12 (m, 4H, $\left.\mathrm{OCH}_{2}\right), 7.41-7.55$ (m, 10H, Ar-H); ${ }^{13} \mathrm{C} \mathrm{NMR}$ $\left(100 \mathrm{MHz}, \mathrm{CDCl}_{3}\right): \delta=26.6,30.1(\mathrm{~d}, J=26.1), 33.7$ (d, $\left.J=27.0\right), 46.7,50.9,66.8,66.9,89.5(\mathrm{~d}$, $J=170.2 \mathrm{~Hz}), 128.2,128.3,128.4,128.5,135.5,136.3,155.4,171.9 .{ }^{19} \mathrm{~F} \mathrm{NMR}\left(100 \mathrm{MHz}, \mathrm{CDCl}_{3}\right)$ : $\delta=-174.5$; MS (ESI, pos) $\mathrm{m} / \mathrm{z}=386$ [M+1]; Anal. Calcd. for $\mathrm{C}_{22} \mathrm{H}_{24} \mathrm{FNO}_{4}: \mathrm{C}, 68.56 ; \mathrm{H}, 6.28 ; \mathrm{N}$, 3.63. Found: C, 68.15; H, 5.95; N, 3.28.

\section{References}

1. a) Wang, J.; Sánchez-Roselló, M.; Aceña, J. L.; del Pozo, C.; Sorochinsky, A. E.; Fustero, S.; Soloshonok, V. A.; Liu, H. Chem. Rev. 2014, 114, 2432; b) Zhou, Y.; Wang, Y.; Gu, Z.; Wang, S.; Zhu, W.; Acena, J. L.; Soloshonok, V. A.; Izawa, K.; Liu, H. Chem. Rev. 2016, 116, 442; c) Fluorine in Pharmaceutical and Medicinal Chemistry: From Biophysical Aspects to Clinical Applications, Imperial College Press, London 2012. Edited by Gouverneur, V. and Müller, K.

2. a) Liang, T.; Neumann, C. N.; Ritter, T. Angew. Chem. Int. Ed. 2013, 52, 8214; b) Campbell, M. G.; Ritter, T. Chem. Rev. 2015, 115, 612; c) Charpentier, J.; Früh, N.; Togni, A. Chem. Rev. 2015, 115, 650; d) Yang, X.; Wu, T.; Phipps, R. J.; Toste, F. D. Chem. Rev. 2015, 115, 826; e) Cresswell, A. J.; Davies, S. G.; Roberts, P. M.; Thomson, J. E. Chem. Rev. 2015, 115, 566; f) O’Hagan, D.; Deng, H. Chem. Rev. 2015, 115, 634; g) Liu, X.; Xu, C.; Wang, M.; Liu, Q. Chem. Rev. 2015, 115, 683; h) Champagne, P. A.; Desroches, J.; Hamel, J. D.; Vandamme, M.; Paquin, J. F. Chem. Rev. 2015, 115, 9073.

3. a) Kiss, L.; Fülöp, F. The Chem. Rec. 2017, doi: 10.1002/tcr201700038; b) Kiss, L.; Nonn, M.; Sillanpää, R.; Fustero, S.; Fülöp, F. Beilstein J. Org. Chem. 2013, 9, 1164; c) Kiss, L.; Nonn, M.; Forró, E.; Sillanpää, R.; Fustero, S.; Fülöp, F. Eur. J. Org. Chem. 2014, 4070. 
4. for some relevant references see: a) Nonn, M.; Kiss, L.; Haukka, M.; Fustero, S.; Fülöp, F. Org. Lett. 2015, 17, 1074; b) Kiss, L.; Nonn, M.; Sillanpää, R.; Haukka, M.; Fustero, S.; Fülöp, F. Chem. Asian J. 2016, 11, 3376; c) Remete, A. M.; Nonn, M.; Fustero, S.; Fülöp, F.; Kiss, L: Molecules 2016, 21, 1943.

5. a) Kiss, L.; Fülöp, F. Chem. Rev. 2014, 114, 1116; b) Kiss, L.; Mándity, I. M.; Fülöp, F. Amino Acids 2017, 49, 1441.

6. a) Kiss, L.; Szatmári, I.; Fülöp, F. Lett. Org. Chem. 2006, 3, 463; b) Kiss, L.; Forró, E.; Fustero, S.; Fülöp, F. Org. Biomol. Chem. 2011, 9, 6528; c) Kiss, L.; Forró, E.; Fustero, S.; Fülöp, F. Eur. J. Org. Chem. 2011, 4993.

7. a) Kiss, L.; Forró, E.; Fülöp, F. Tetrahedron 2012, 68, 4438; b) Kiss, L.; Forró, E.; Martinek, T. A.; Bernáth, G.; De Kimpe, N.; Fülöp, F. Tetrahedron 2008, 64, 5036.

\section{Supporting Information. NMR spectra of the synthetized compounds}



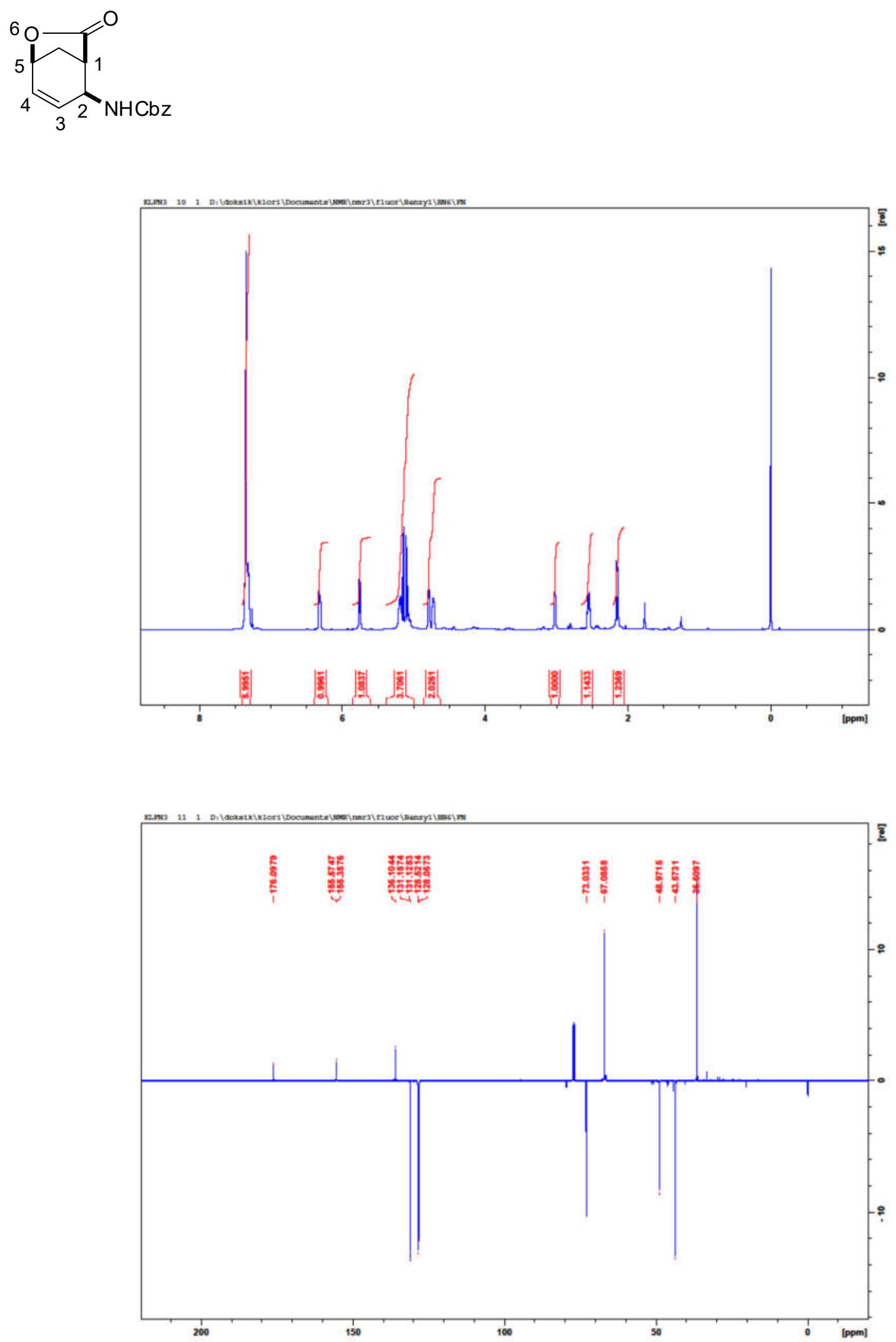

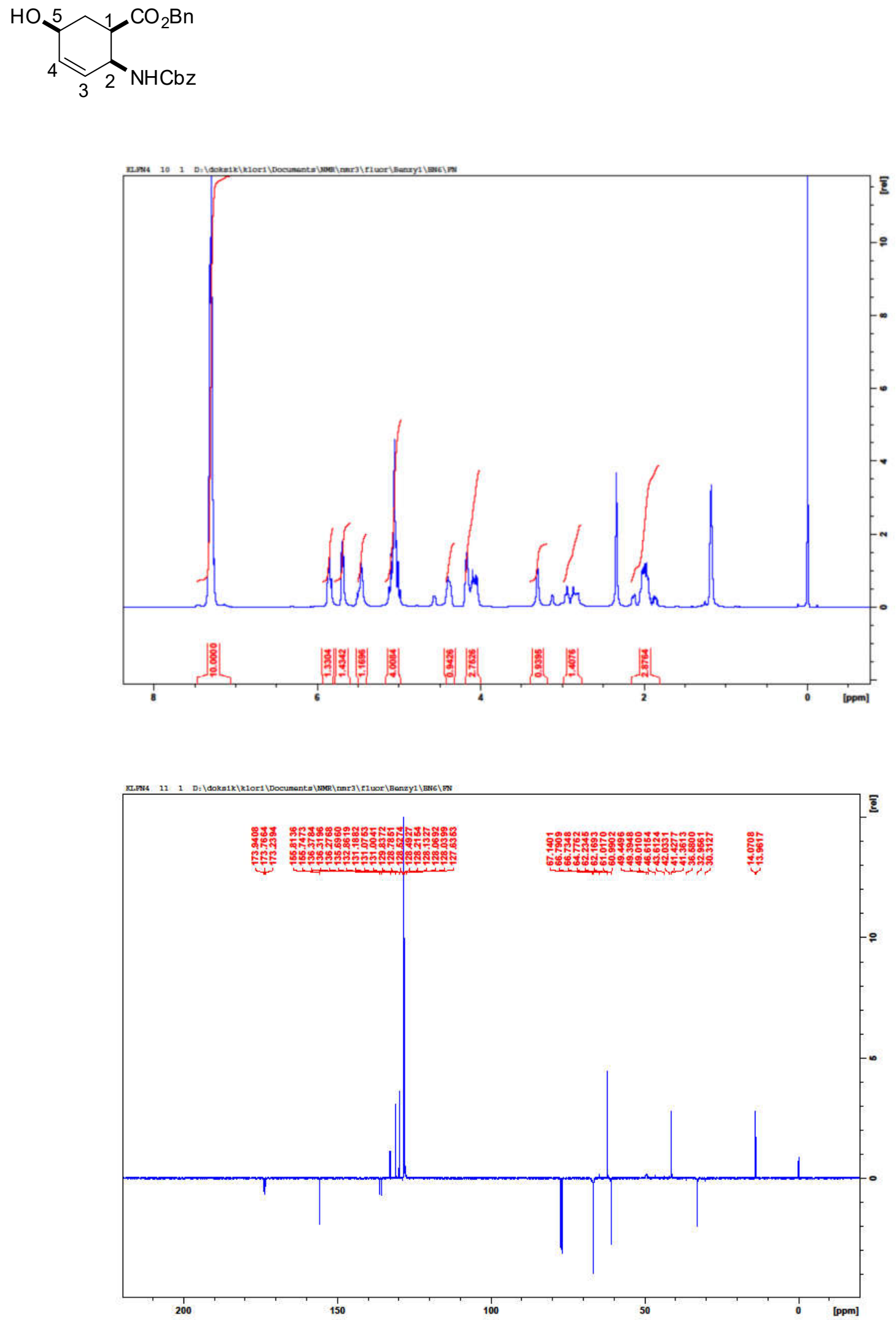


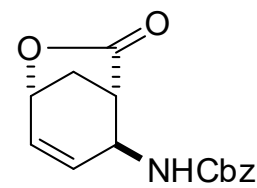
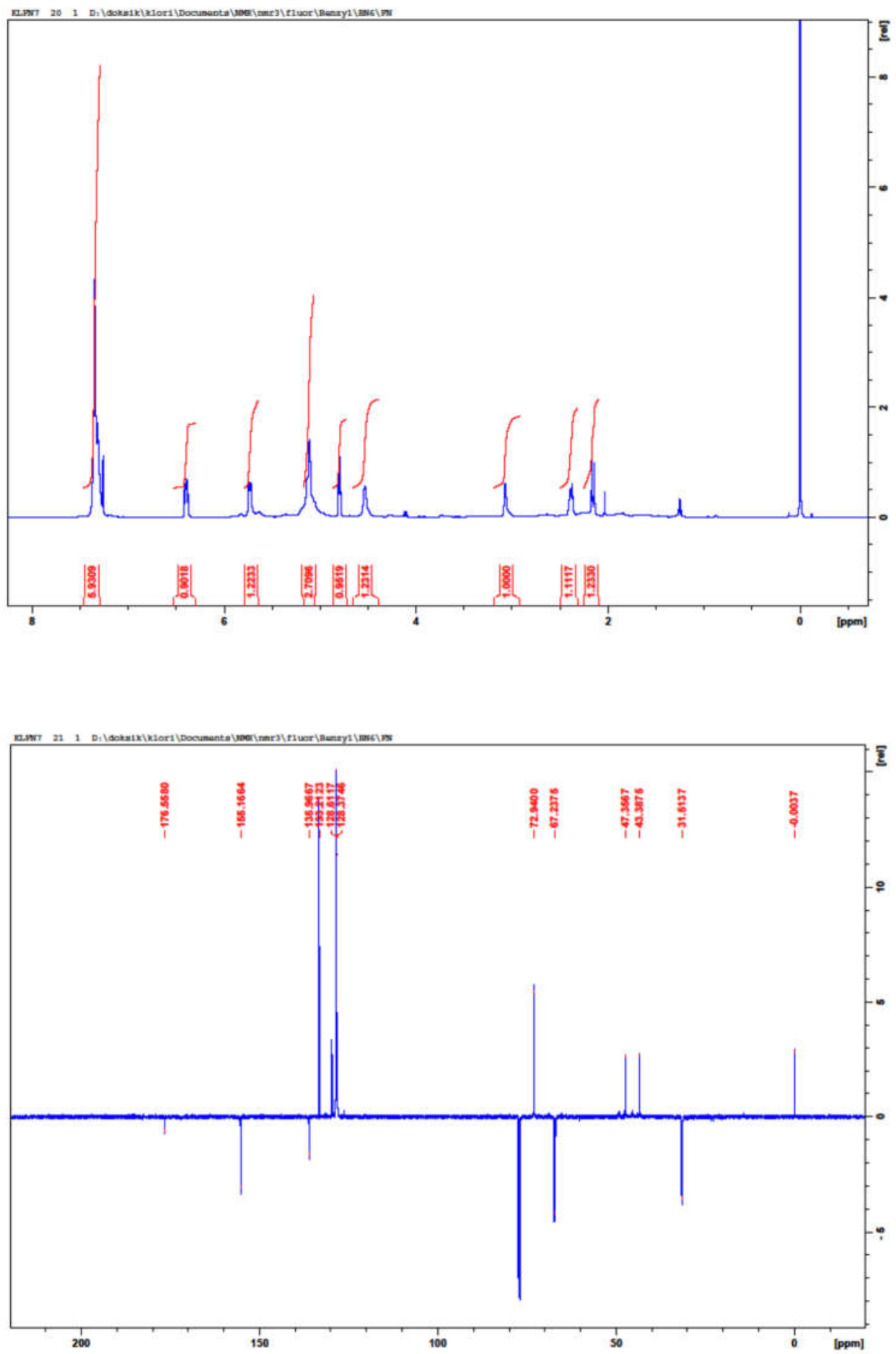
<smiles>O=C(NC1C=C[C@@H](O)C[C@H]1C(=O)OCc1ccccc1)OCc1ccccc1</smiles>
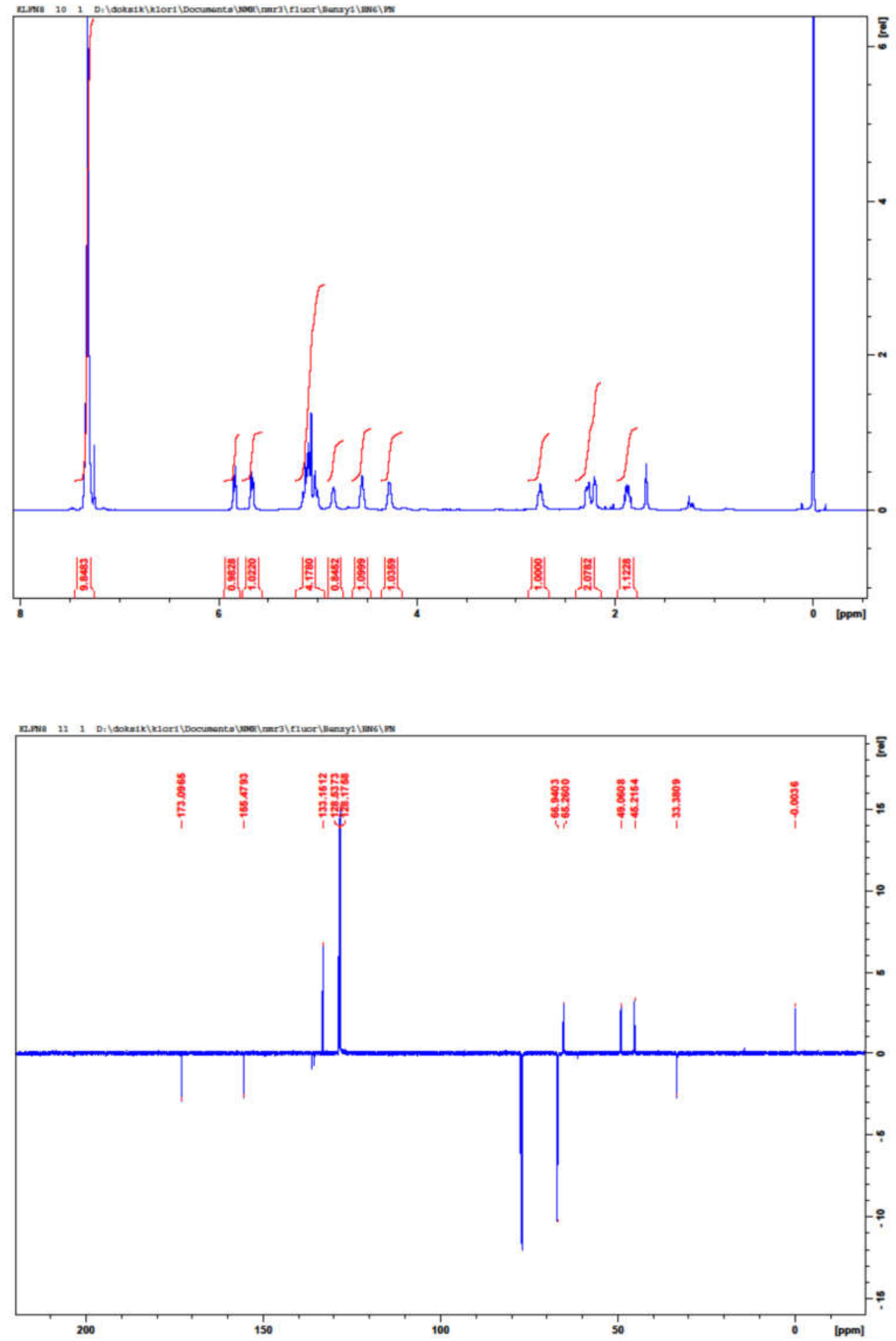
Online journal "Fluorine notes" ISSN 2071-4807, Vol. 1(116), 2018; DOI: 10.17677/fn20714807.2018.01.01<smiles>CC(=O)NC1C=CC(F)C[C@H]1C(=O)OCc1ccccc1</smiles>
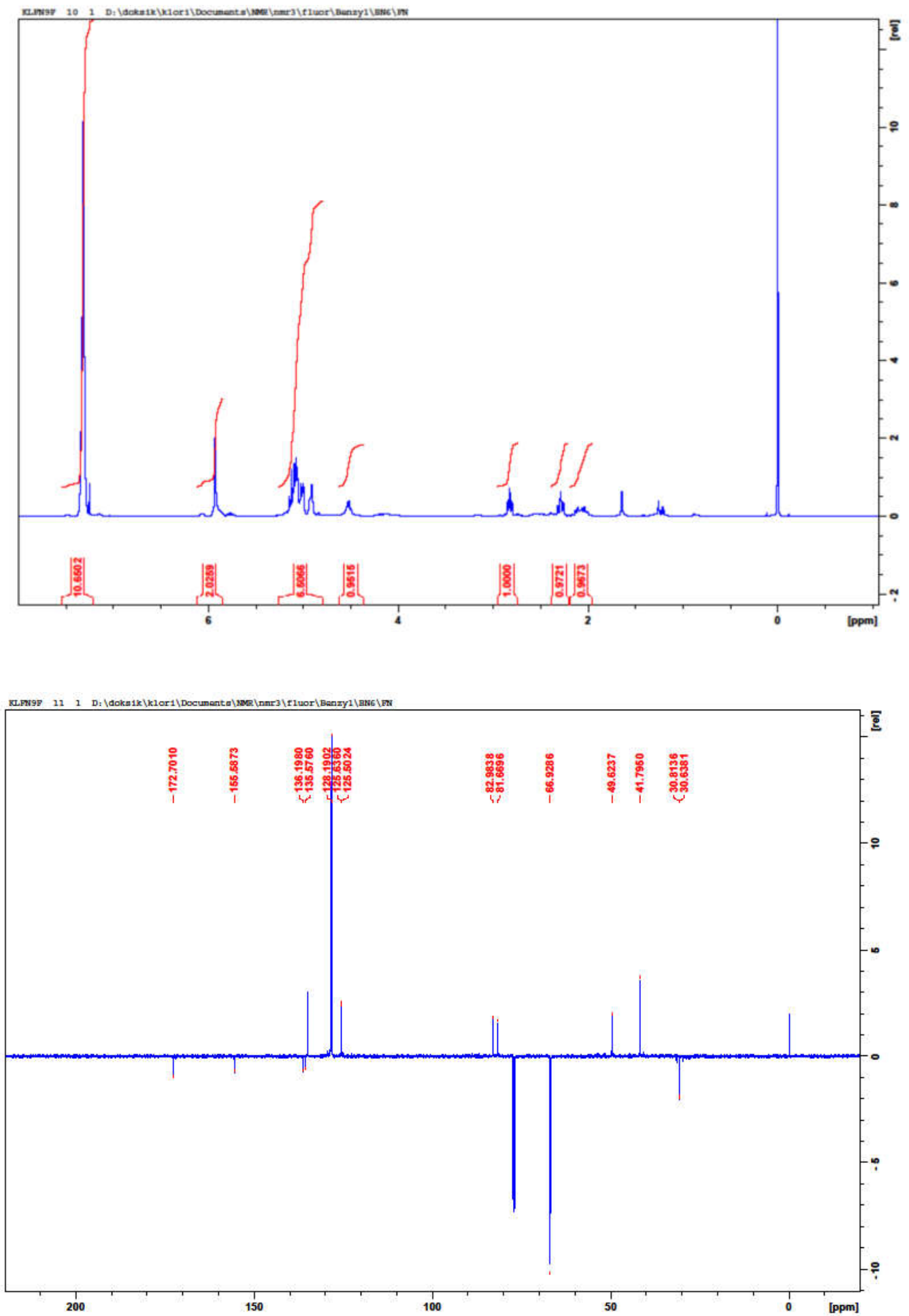
<smiles>CC(=O)NC1CC=CCC1C(=O)OCc1ccccc1</smiles>
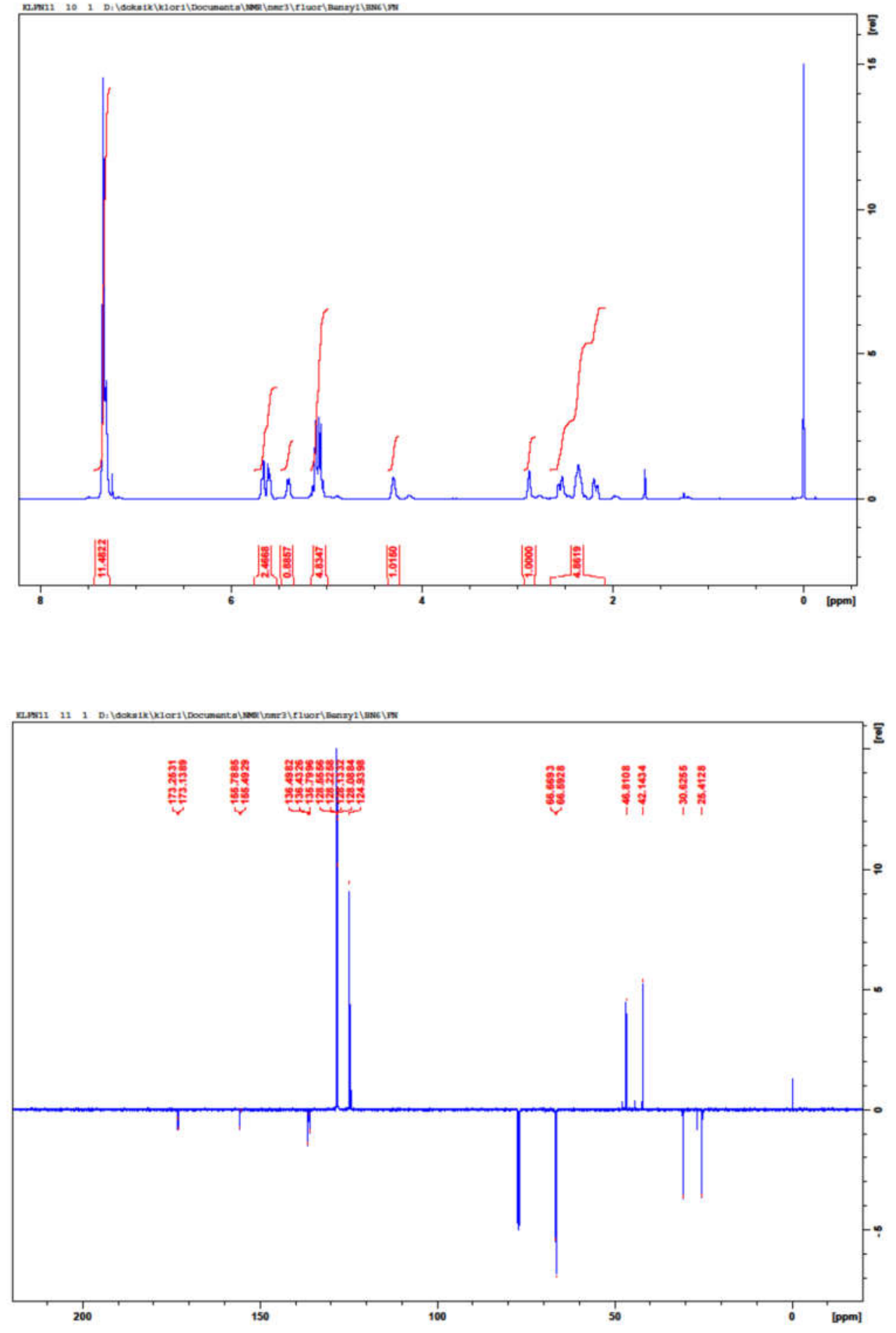
<smiles>CC(=O)NC1CC2OC2CC1C(=O)OCc1ccccc1</smiles>
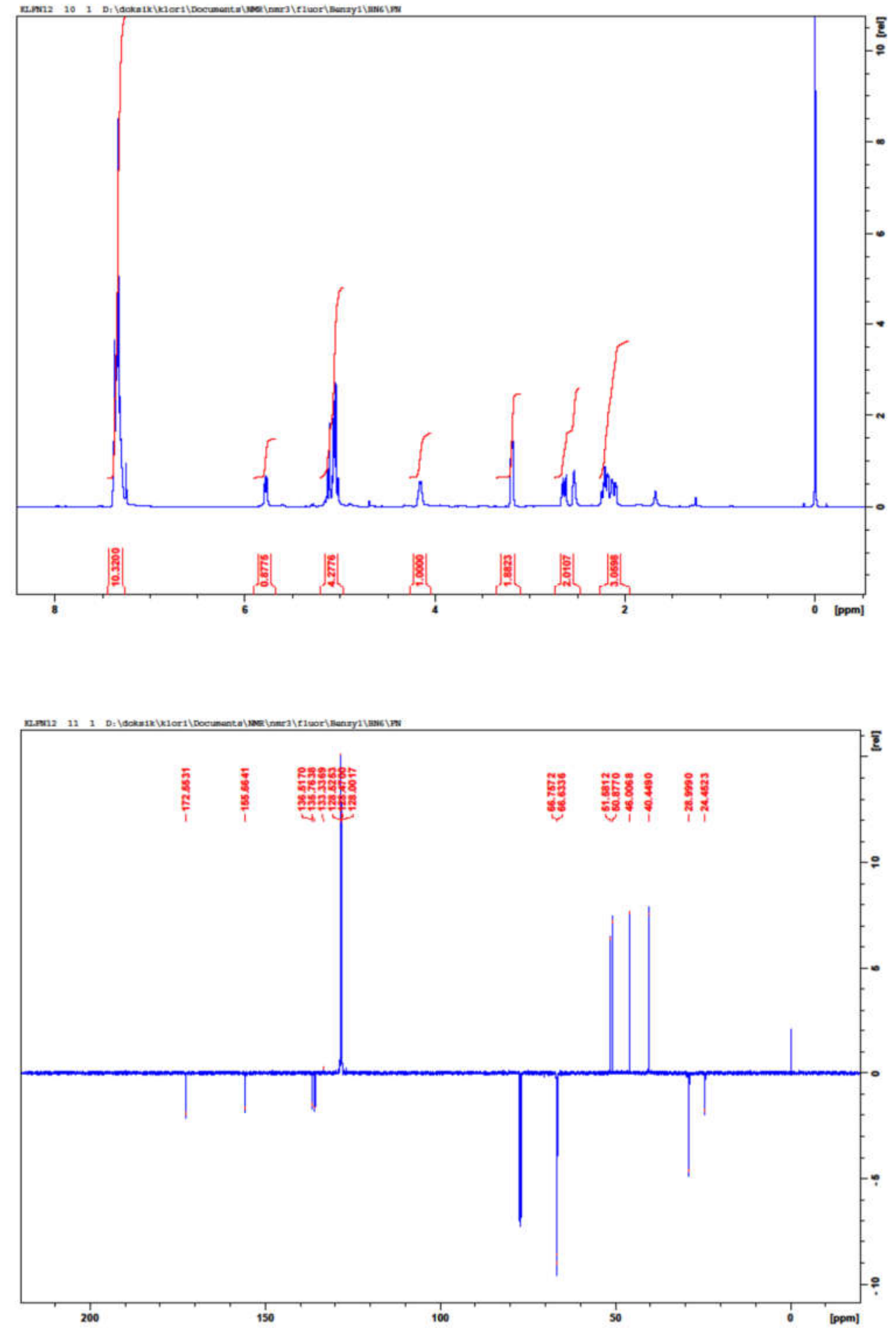
<smiles>O=C(NC1CC(O)CCC1C(=O)OCc1ccccc1)c1ccccc1</smiles>
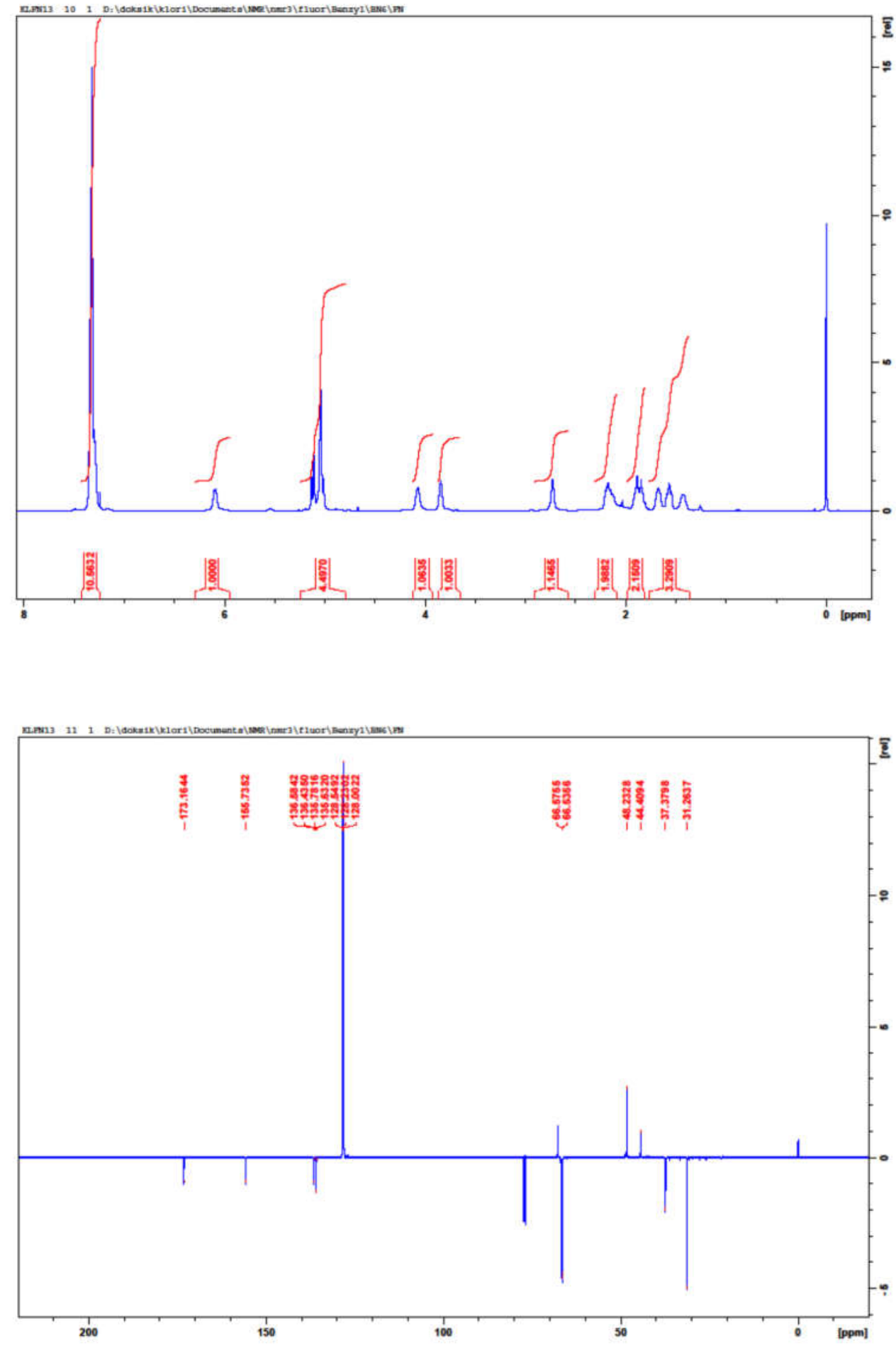
<smiles>CC(=O)NC1CC=CC[C@H]1C(=O)OCc1ccccc1</smiles>
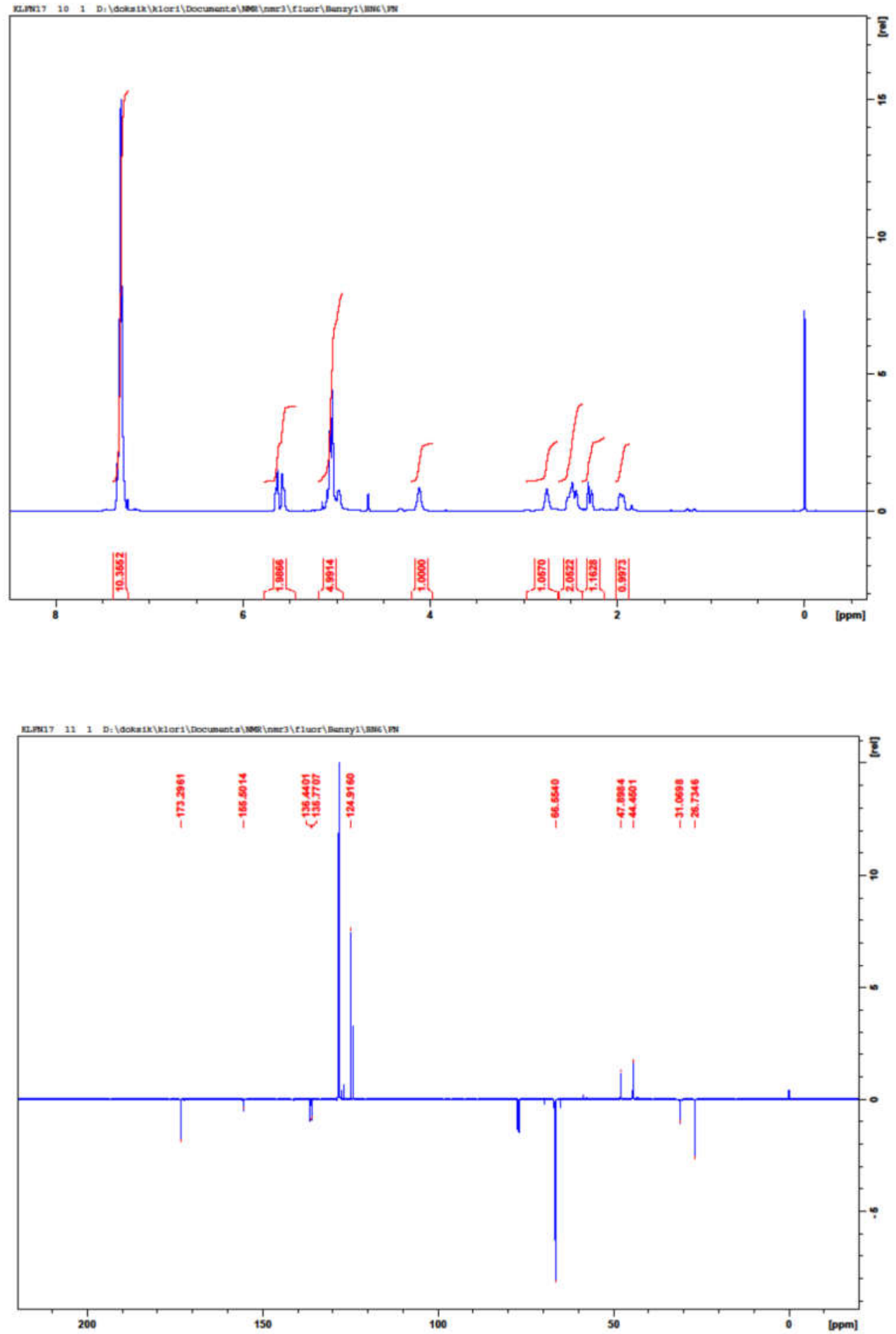
<smiles>O=C(OCc1ccccc1)C1CC2OC2C1</smiles>
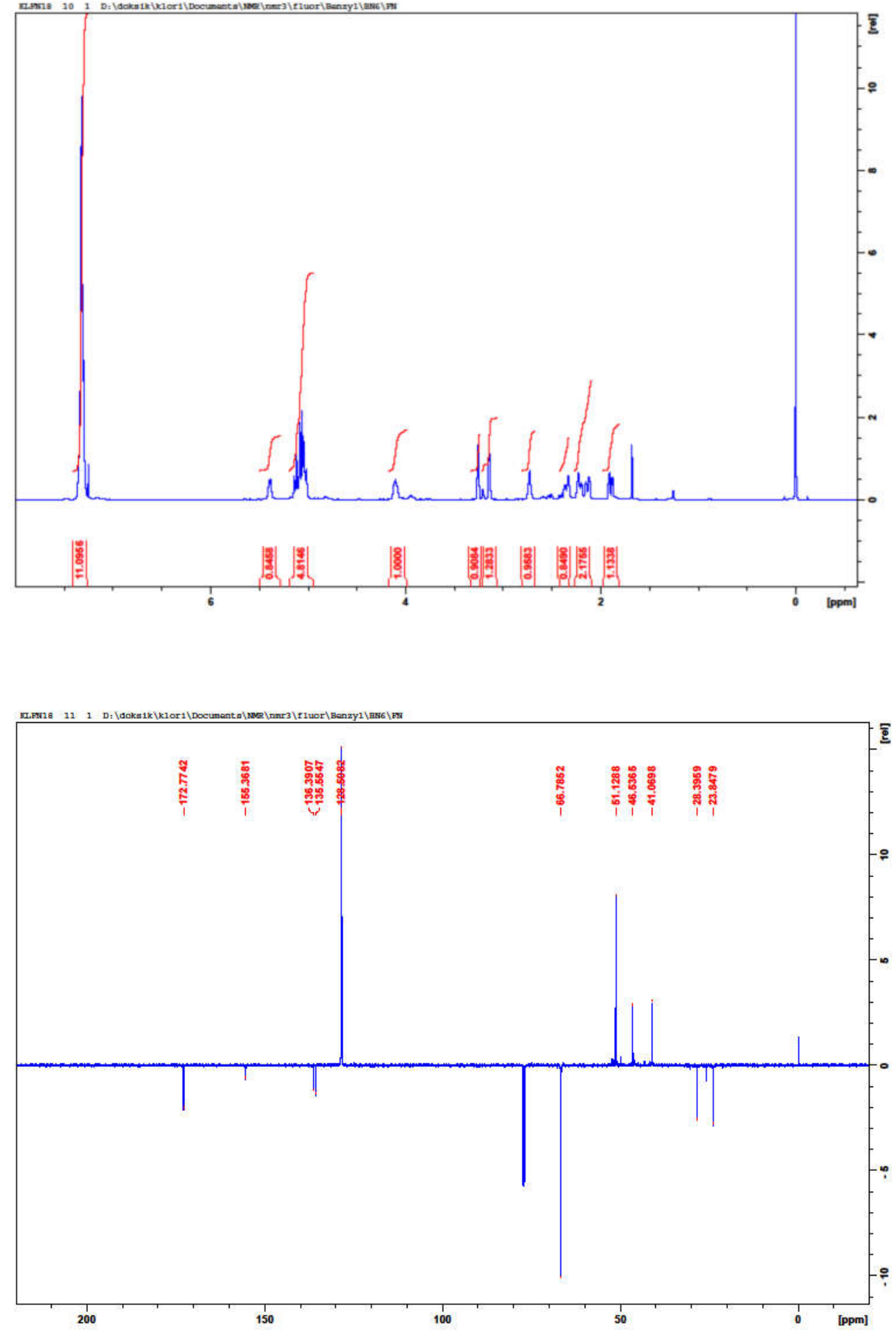
<smiles>[R]OC(=O)NC1CCC(O)C[C@H]1C(=O)OCc1ccccc1</smiles>
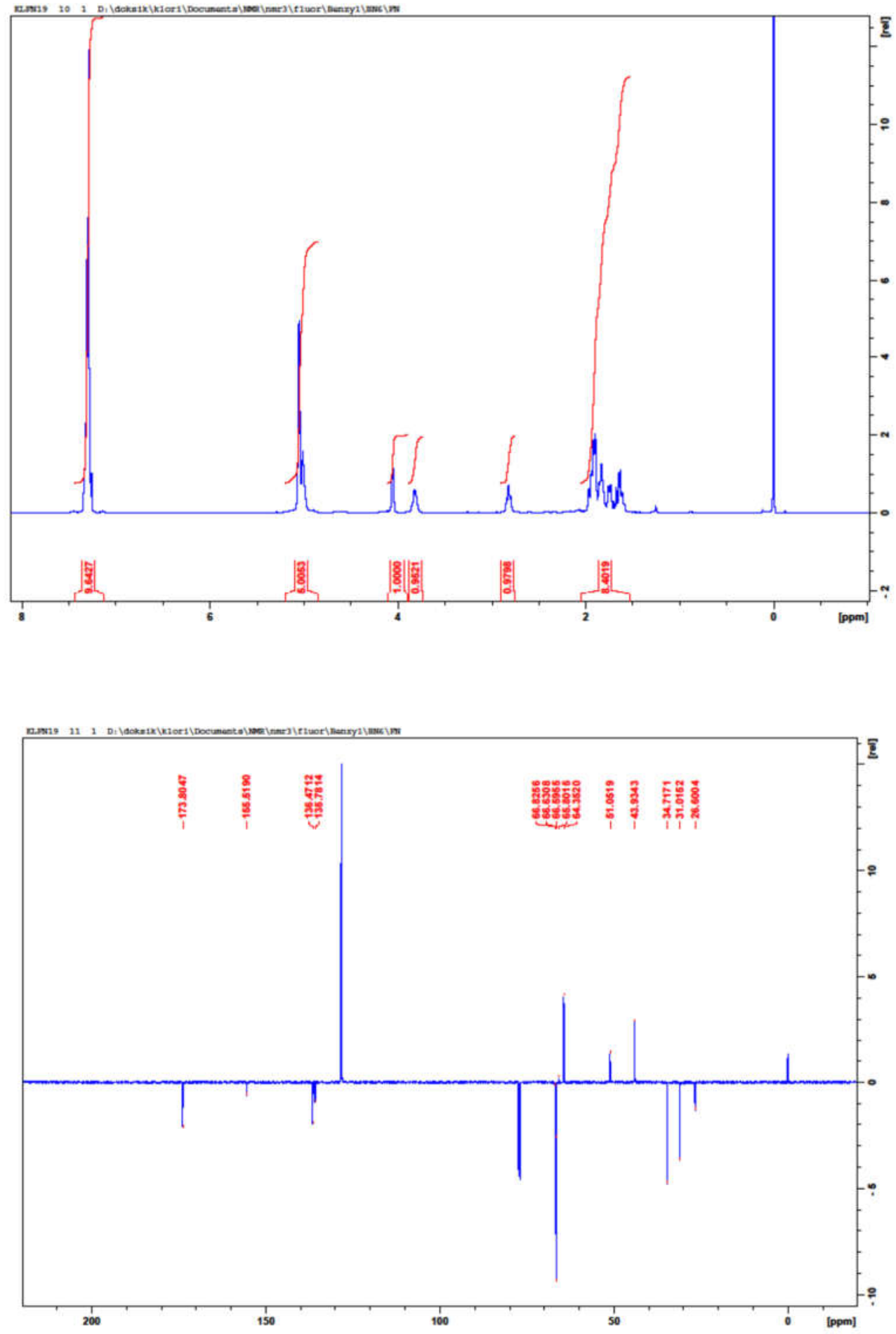
<smiles>O=C(NC1CC[C@@H](F)C[C@H]1C(=O)OCc1ccccc1)OCc1ccccc1</smiles>
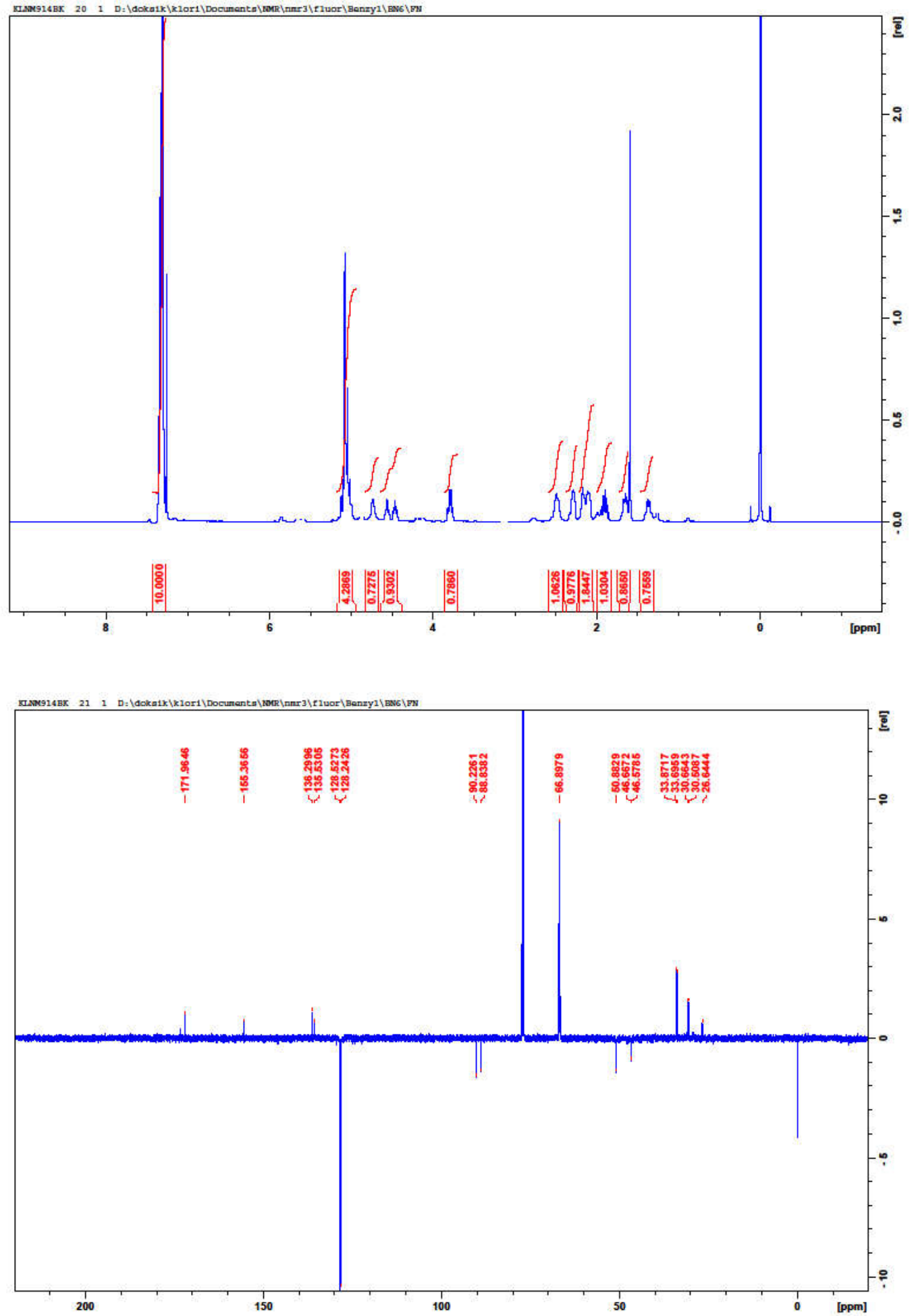

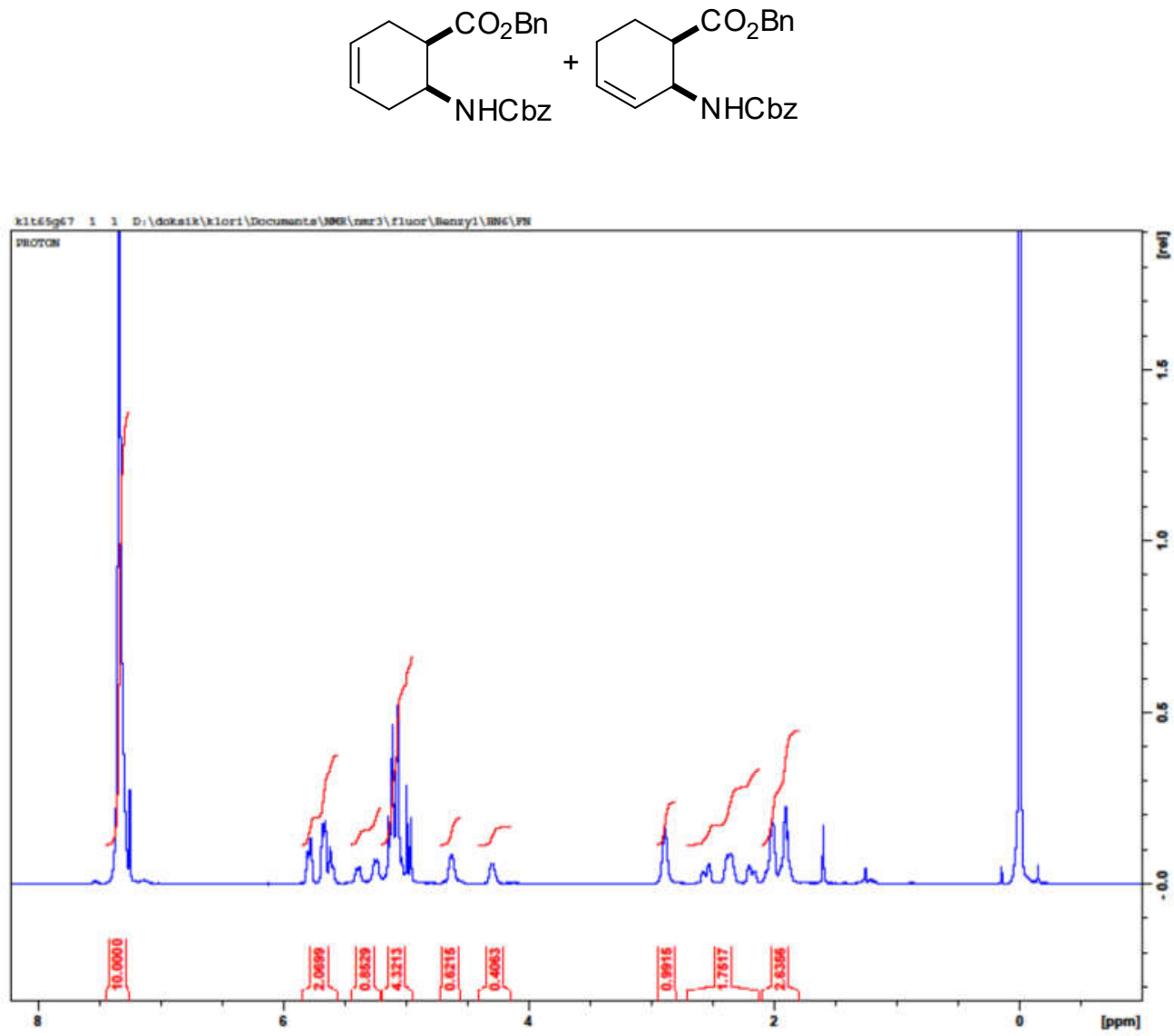